\title{
Jean-Philippe Beaulieu, Diane Desrosiers-Bonin, a cura di, Hélisienne de Crenne. L'écriture et ses doubles
}

\section{Filippo Fonio}

\section{Q OpenEdition}

10 Journals

\section{Édition électronique}

URL : http://journals.openedition.org/studifrancesi/34496

DOI : $10.4000 /$ studifrancesi.34496

ISSN : 2421-5856

Éditeur

Rosenberg \& Sellier

\section{Édition imprimée}

Date de publication : 1 novembre 2005

Pagination : 400-401

ISSN : 0039-2944

\section{Référence électronique}

Filippo Fonio, « Jean-Philippe Beaulieu, Diane Desrosiers-Bonin, a cura di, Hélisienne de Crenne. L'écriture et ses doubles », Studi Francesi [En ligne], 146 (XLIX | II) | 2005, mis en ligne le 30 novembre 2015, consulté le 20 avril 2021. URL : http://journals.openedition.org/studifrancesi/34496 ; DOI : https://doi.org/10.4000/studifrancesi.34496

Ce document a été généré automatiquement le 20 avril 2021.

\section{(c)}

Studi Francesi è distribuita con Licenza Creative Commons Attribuzione - Non commerciale - Non opere derivate 4.0 Internazionale. 


\title{
Jean-Philippe Beaulieu, Diane Desrosiers-Bonin, a cura di, Hélisienne de Crenne. L'écriture et ses doubles
}

\author{
Filippo Fonio
}

\section{RÉFÉRENCE}

JEAN-PHILIPPE BEAULIEU, DIANE DESROSIERS-BONIN, a cura di, Hélisienne de Crenne. L'écriture et ses doubles, Paris, Honoré Champion («Études et essais sur la Renaissance», 54), 2004, pp. 292.

1 Les CEuvres (1543) di Hélisienne de Crenne, alias Marguerite Briet, rappresentano una tappa importante per lo sviluppo delle forme narrative in Francia nella prima metà del Cinquecento. Il volume, realizzato con la collaborazione dell'équipe GARSE-XVI (Groupe d'Analyse et de Recherche sur l'Écriture féminine au XVI siècle, McGill University Université de Montréal), raccoglie recenti contributi consacrati alla sua opera. In particolare, gli interventi sono incentrati sull'azione delle dinamiche della ripresa e dell'intertestualità nelle Angoysses douloureuses qui procedent d'amours (1538), nelle Epistres familieres et invectives (1539) e nel Songe de Madame Helisenne (1540), testi che costituiscono il corpus dell'autrice, oltre a una traduzione dei primi quattro libri dell'Eneide (1541). Ad apertura del volume, una presentazione dei curatori traccia le linee della fortuna di Hélisienne de Crenne (JEAN-PHILIPPE BEAULIEU, DIANE DESROSIERS-BONIN, Les jeux du même, de l'autre et du multiple chez Hélisienne de Crenne, pp. 9-17).

2 La prima serie dei contributi qui raccolti è dedicata al roman sentimental Les Angoysses douloureuses qui procedent d'amours, letto anzitutto a partire dal tessuto retorico dell'opera e dalle implicazioni religiose e giudiziarie della rhétorique de l'aveu: VIRGINIA KRAUSE, Confessions d'une héroïne romanesque: les Angoysses douloureuses d'Hélisienne de 
Crenne, pp. 19-34. La studiosa individua una serie di analogie fra il paradigma d'amore e quello della confessione, evidenziando come Hélisienne tenti di rivelare lo scacco della logica dell'esempio, codice impersonale e attanziale, stravolgendo i canoni di una narrativa intesa come finzione esemplare. JANINE INCARDONA si occupa della funzione dei ritratti femminili nelle Angoysses in quanto mezzi per la costruzione di un'identità, considerando la parentela con gli stilemi dei blasons e dei contre-blasons, nonché le strategie tematiche, strutturali e stilistiche alla base dei ritratti (Jeux de portraits dans Les Angoysses douloureuses qui procedent d'amours, pp. 35-53). Particolare attenzione è dedicata alle dinamiche della riflessione e del moltiplicarsi del punto di vista, nel passaggio dalla prima alla seconda parte delle Angoysses, in cui alla voce narrante di Hélisienne subentra quella di Guenelic, figura che conserva tracce dei caratteri dell'hérö̈ne tragique (CATHLEEN M. BAUSCHATZ, Travestissement textuel dans la 'Seconde partie' des Angoysses douloureuses, pp. 55-70), o laddove l'identità viene costruita attraverso la scrittura, mediante alcune metafore significative quali il combattimento spirituale o lo specchio (CHRISTINE DE BUZON, Les miroirs d'Hélisienne de Crenne, pp. 71-81). La matrice delle metafore tratte dal linguaggio musicale, che nell'opera di Hélisienne paiono inserirsi in una concezione musicale dell'esperienza narrativa stessa, in vista appunto della creazione di una 'musica narrativa', sono oggetto del contributo di CATHARINE RANDALL, 'L'argent vif' dedans la statue mobile: musique, mouvement et mutabilité chez Hélisienne de Crenne, pp. 123-130. mARTINE DEBAISIEUX (La prison du désir: Hélisienne de Crenne et Théophile de Viau, pp. 111-121) si occupa di una serie di analogie fra le Angoysses e i motivi dell'imprigionamento, del prezzo della libertà amorosa e dell'ambivalenza nella rappresentazione del desiderio, costanti dell'autore degli Amours tragiques de Pyrame et Thisbé. A chiudere questa prima sezione dedicata alle Angoysses, MARIE CLAUDE MALENFANT propone uno studio relativo alla non-univocità del percorso didattico dell'opera, caratterizzata dalla presenza di un'eroina al contempo esemplare e nonesemplare, nonché da un ricorso costante a una dinamica del raddoppiamentoribaltamento data da una ricorrenza dissimilare nelle serie iterative: Quelques modalités exemplaires des Angoysses douloureuses qui procedent d'amours d'Hélisienne de Crenne (1538), pp. 83-109.

Mentre il contributo di DIANE S. WOOD, Les lettres d'Hélisienne de Crenne et Jacopo Caviceo: lecture et stratégies scripturaires (pp. 131-152) individua l'influenza di Jacopo Caviceo tanto sulla componente epistolare delle Angoysses quanto sulle Epistres di Hélisienne, i successivi tre studi sono incentrati specificamente sulla seconda opera di Marguerite Briet, le Epistres appunto. JERRY C. NASH analizza il ruolo strategico dell'impiego della prima persona e del modo performativo nella raccolta, come tentativo di appropriarsi di una virilità letteraria (Discours performatif d'une femme écrivain 'exerceant cuvres viriles': Les Epistres familieres et invectives d'Hélisienne de Crenne, pp. 153-167). Inventio, dispositio ed elocutio nelle Epistres sono invece oggetto dello studio di LUC VAILLANCOURT, Les Epistres familieres et invectives de ma dame Helisienne: 'contentio orationis' ou 'sermo pedestris'?, pp. 169-195. Lo studioso inquadra l'opera di Hélisienne nell'ambito della tradizione dell'epistolografia morale, genere praticato fra gli altri da Jean Bouchet, individuando la relativamente scarsa incidenza dei topoi della scrittura epistolare e la maggiore prossimità delle Epistres agli stilemi della contentio più che a quelli del sermo petrarchesco e ciceroniano. COLETTE H. WINN, infine, legge le Epistres come crocevia di influenze antiche e moderne nella scrittura d'amicizia: 'Ce lien si ferme et si puissant...'. 
'Amicitia' et 'consolatio' dans les «Epistres familieres» d'Hélisienne de Crenne (1539), pp. 197-215.

4 Due contributi sono dedicati al Songe di Hélisienne: ROBERT D. COTTRELL (Le Songe d'Hélisienne de Crenne, pp. 217-240) considera la componente spirituale dell'opera e la scelta del genere come elementi che denotano un desiderio di inclusione nei circoli umanistici dell'epoca; DIANE DESROSIERS-BONIN (L'architecture exégétique du Songe d'Hélisienne de Crenne, pp. 241-250) legge l'opera alla luce delle formulazioni dantesche della poetica dell'allegoria e della pratica interpretativa dell'allegoresi.

5 JEAN-PHILIPPE BEAULIEU considera l'opera di Hélisienne come caratterizzata nel complesso da una struttura tripartita, tanto a livello infra-testuale quanto sul piano di una valutazione del corpus dell'autrice (Tripartitions dans l'œuvre d'Hélisienne de Crenne, pp. 251-263).

6 Seguono una Bibliografia delle edizioni antiche e moderne di Hélisienne e della critica, e un index nominum. 